\title{
Dural sinus thrombosis identified by point-of-care ultrasound
}

eISSN: 2383-4625

\author{
Laura T. Director, David C. Mackenzie \\ Department of Emergency Medicine, Maine Medical Center, Portland, ME, USA
}

Dural sinus thrombosis (DST), or cerebral venous thrombosis, is an uncommon cause of stroke. It has a variable presentation, and the symptoms and signs can be non-specific. The diagnosis of DST can be difficult to make and is often delayed or missed. Computed tomography venography or magnetic resonance venography are the typical imaging modalities used to diagnose DST. However, computed tomography venography and magnetic resonance venography both have limitation for emergency department patients. In this article, we report the use of point-of-care ultrasound to facilitate the diagnosis of DST.

Keywords Diagnostic imaging; Thrombosis; Stroke; Emergencies

Capsule
Wummary is already known
$\begin{aligned} & \text { Diagnosis of dural sinus thrombosis in emergency department patients is diffi- } \\ & \text { cult and frequently delayed. Advanced imaging is typically required to evaluate } \\ & \text { for a dural sinus thrombosis. }\end{aligned}$
$\begin{aligned} & \text { What is new in the current study } \\ & \text { We report a novel use of point-of-care ultrasound to facilitate the diagnosis of } \\ & \text { a dural sinus thrombosis. }\end{aligned}$

\section{INTRODUCTION}

Dural sinus thrombosis (DST) is a rare yet potentially fatal disease. DST has a variable presentation and its non-specific symptoms can make the identification of the condition challenging. While advances in imaging techniques have facilitated a faster recognition of DST, delays to diagnosis and misdiagnosis remain common. Prompt recognition and treatment can improve patient outcomes, making early identification of the disease critical. ${ }^{1,2}$

The presenting symptoms and signs of DST vary with the location and size of the thrombus. Patients most commonly complain of a severe headache. They can also present with encephalopathy, fever, seizures, or neurologic deficits. DST can progress rapidly, resulting in neurologic deterioration and death. ${ }^{3}$

We report a case of a patient presenting with unilateral headache and neck pain, in which the
Received: 11 May 2017

Revised: 14 June 2017

Accepted: 20 June 2017

Correspondence to: David C. Mackenzie Department of Emergency Medicine, Maine Medical Center, 22 Bramhall Street, Portland, ME 04102, USA E-mail: DMackenzie@mmc.org

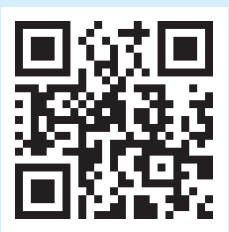

How to cite this article:

Director LT, Mackenzie DC. Dural sinus thrombosis identified by point-of-care ultrasound. Clin Exp Emerg Med 2018;5(3):199-203.

This is an Open Access article distributed under the terms of the Creative Commons Attribution Non-Commercial License (http:// creativecommons.org/licenses/by-nc/4.0/). 
use of emergency physician-performed point-of-care ultrasound allowed rapid identification of a thrombosis in the patient's internal jugular vein, with intracranial extension consistent with DST. Anticoagulation was initiated rapidly and the diagnosis confirmed with computed tomography angiography. This case illustrates a novel use of point-of-care ultrasound to help detect DST.

\section{CASE REPORT}

A 33-year-old female presented to the emergency department (ED) with a one-week history of global headache, sinus pressure, and gradual onset of pain in the right side of the neck. The patient reported nausea, vomiting, photophobia, and a severely worsened unilateral headache for twelve hours prior to presenting to the ED. She had no recent head injury and denied a history of headaches. She had been ill with an upper respiratory infection in the week prior to developing the headache. She had no complaint of weakness, sensory change, or vision loss. On arrival to the ED, her blood pressure was 136/89 $\mathrm{mmHg}$, pulse 67, respiratory rate 16 , temperature $36.5^{\circ} \mathrm{C}$, and pulse oximetry $100 \%$ on room air. Physical examination revealed focal paraspinal neck tenderness at the right craniocervical junction, right anterolateral neck tenderness, and no meningeal signs. Her neurological examination was normal and the rest of the examination was unremarkable. She had no medical or surgical history, and no family history of thromboembolic disease. She did not use tobacco, alcohol, or drugs. Her only medication was oral contraception.

The patient was given intravenous ondansetron and morphine to control her symptoms. Given the history of oral contraceptive use and localized neck pain, the differential diagnosis included deep venous thrombosis, dural sinus thrombosis, and migraine headache. The treating emergency physician performed a pointof-care ultrasound to assess the neck vasculature for thrombosis. Images were obtained using a 5- to $10-\mathrm{MHz}$ linear transducer (SonoSite, Bothell, WA, USA). The probe was placed in a transverse orientation and moved caudal and cephalad until the carotid artery and internal jugular vein were located on the right aspect of the patient's neck. Long and short axis images of the carotid artery and internal jugular vein were obtained. A hyperechoic, non-compressible structure was observed in the internal

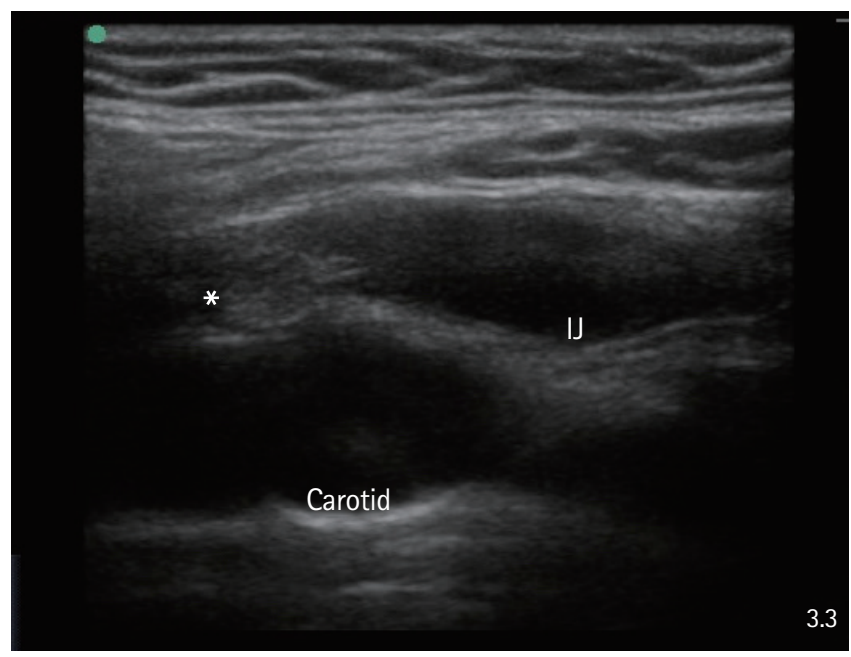

Fig. 1. Ultrasound of the right internal jugular (IJ) vein and carotid artery in long axis, showing thrombosis (asterisk) in the superior aspect of the jugular vein.
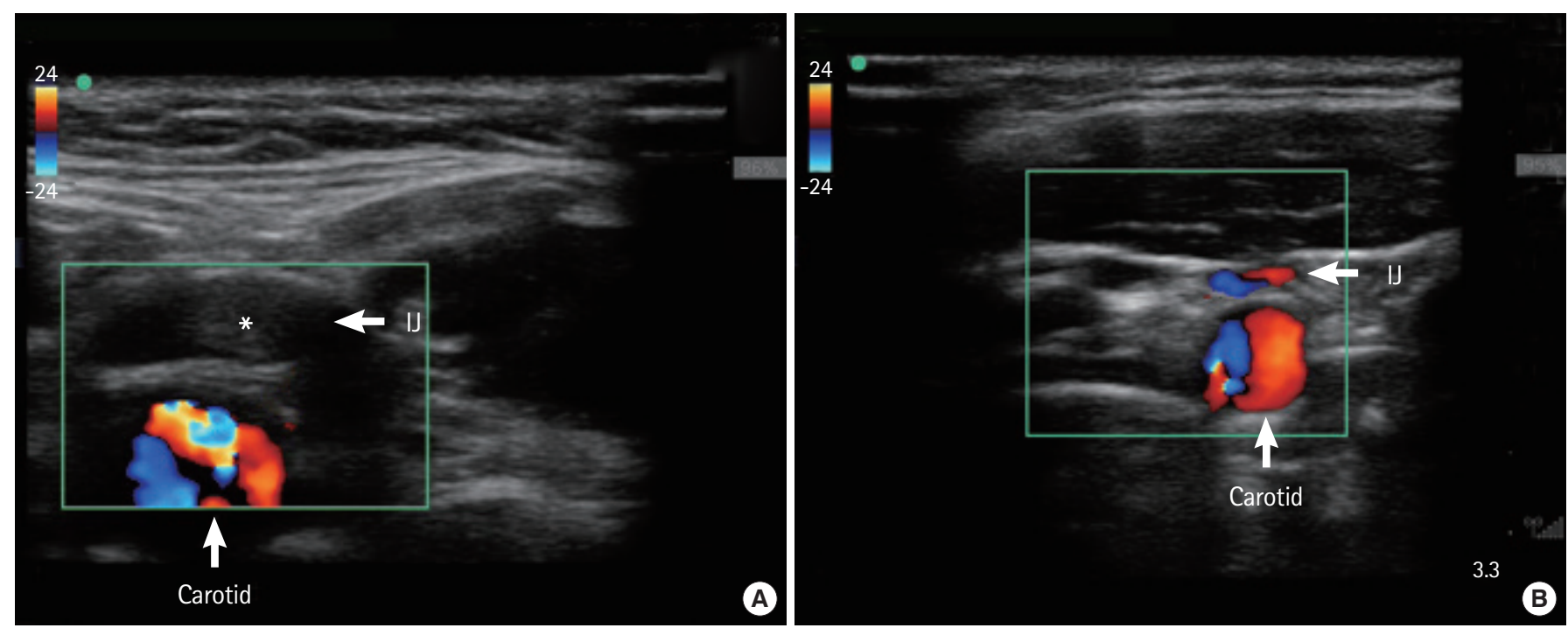

Fig. 2. (A) Ultrasound demonstrating blood flow through carotid artery on patient's right side. (B) On the contralateral side, ultrasound demonstrates blood flow through both the carotid and internal jugular (IJ) vein. Thrombus visualized in patient's right IJ vein (asterisk). 


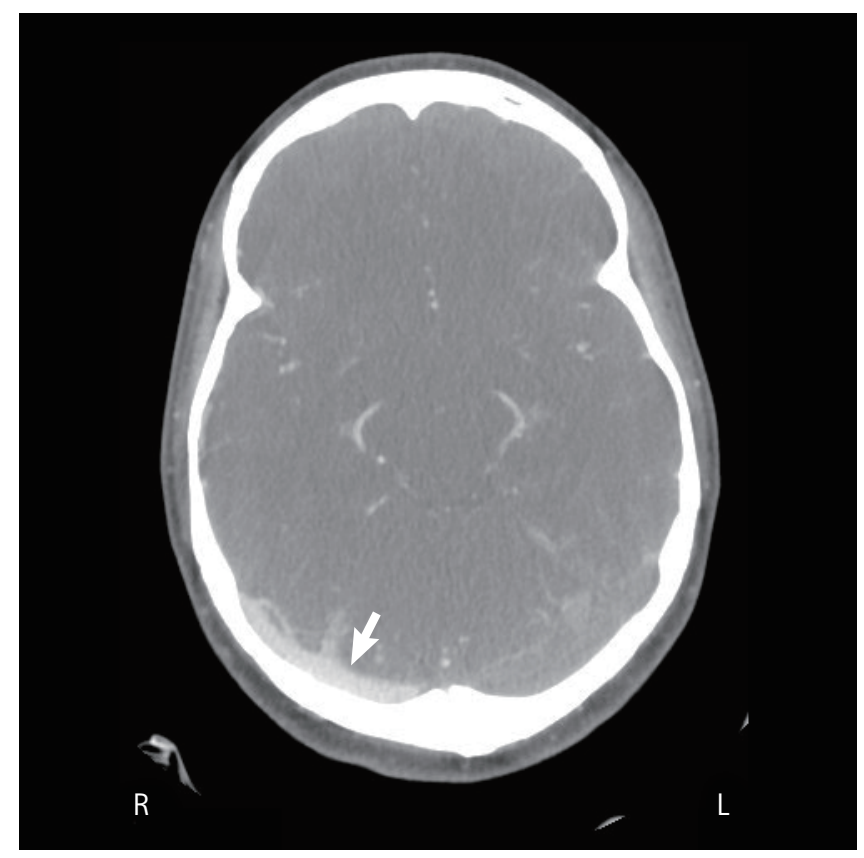

Fig. 3. Computed tomography scan showing region of hyperintensity posterolaterally on patient's right (R) side consistent with thrombus. $\mathrm{L}$, left.

jugular vein, consistent with thrombus (Fig. 1). This lesion was noted to extend cranially as far as the treating physician could acquire an image in both long and short axis of the vessel. There was no extension of the thrombus proximally toward the heart. Color Doppler examination of the vessels demonstrated blood flow in the carotid artery but not in the internal jugular vein on the affected side; the contralateral side showed flow in both the artery and vein (Fig. 2). The finding of thrombus in the right jugular vein with intracranial extension prompted the diagnosis of a dural sinus thrombosis. Treatment with high intensity intravenous heparin was initiated.

Computed tomography (CT) angiography and CT venography (CTV) of the head and neck was subsequently obtained to confirm the point-of-care ultrasound findings, and to evaluate the extent of clot burden. This revealed an area of hyperintensity in the right transverse sinus of the right parieto-occipital lobe (Fig. 3), confirming the presence of thrombosis. The thrombus extended from the undersurface of the parieto-occipital lobe into the right internal jugular vein to the level of the hyoid bone, consistent with the point-of-care ultrasound findings (Fig. 4). There was no evidence of free fluid, infarct, edema, or intra-axial or extra-axial hemorrhage. The CT imaging was otherwise unremarkable.

The patient was admitted to the intensive care unit for ongoing care. Oral anticoagulation with warfarin was started. A repeat computed tomography angiography twenty-four hours after ad-

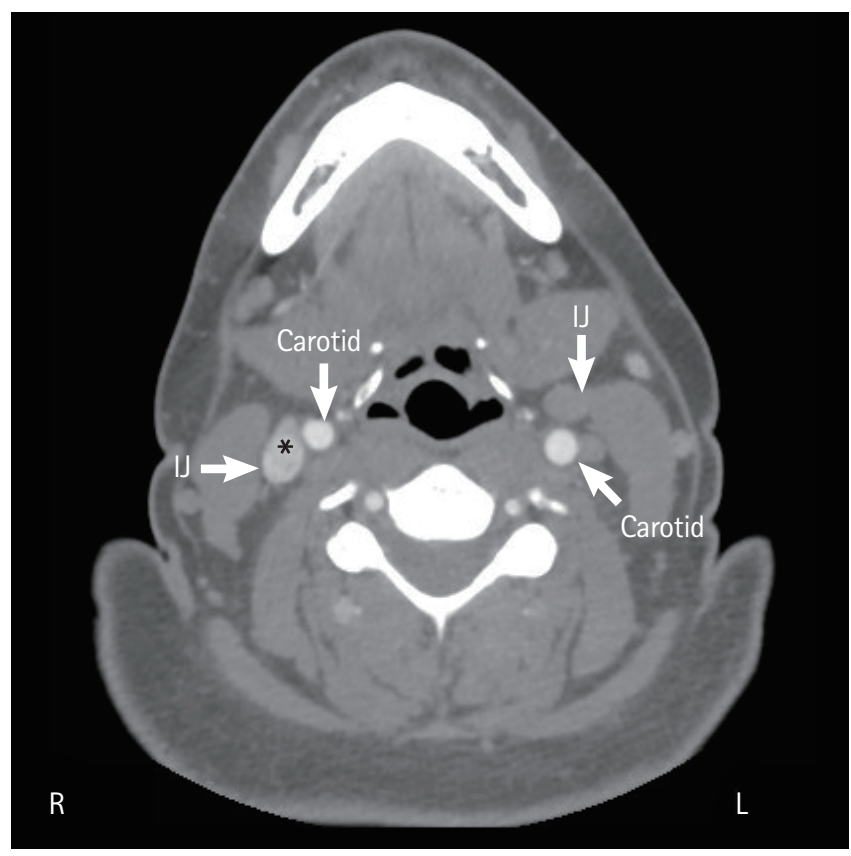

Fig. 4. Computed tomography scan results show a region of hyperintensity (asterisk) in right (R) internal jugular (IJ) vein compared to contralateral IJ vein. $L$, left.

mission showed a stable appearance of the thrombosis in the transverse sinus. On the third hospital day, the patient's symptoms were well controlled, the international normalized ratio was therapeutic, and the patient was discharged to follow up with her primary physician. At follow up she was found to have a pro-thrombotic phenotype and was advised to continue anticoagulation indefinitely.

\section{DISCUSSION}

DST is a rare yet potentially fatal cerebrovascular disease, which primarily affects young adults and children, accounting for approximately $1 \%$ to $2 \%$ of strokes. ${ }^{4}$ DST can progress rapidly and lead to neurological deterioration and death due to cerebral ischemia. Additionally, approximately $30 \%$ of patients develop intracerebral hemorrhage from venous stasis and rupture of the bloodbrain-barrier. ${ }^{5}$ Early identification of the disease is critical to initiating timely therapy and preventing potentially serious complications.

The clinical presentation of DST is highly variable, and the outcome can range from full recovery to death. Patients complain of a severe headache in about $75 \%$ of cases. ${ }^{6}$ The headache can occur alone or in conjunction with symptoms such as fever, lethargy, vomiting, or seizures. Examination findings may range from normal to focal neurologic deficits, vision loss, increased intraoc- 
ular pressure, ptosis, impaired cognition, and coma, depending on the location and severity of the clot burden. ${ }^{7.8}$

Risk factors for DST include prothrombotic conditions, exogenous hormone use, pregnancy, head trauma, malignancy, and infection. ${ }^{9}$ Prothrombotic risk factors are ultimately identified in the majority of patients. ${ }^{4}$ The acute management of DST centers on the prevention of the clot propagation, recanalization of the thrombosed sinus, and preventing associated complications such as infection. ${ }^{10}$ Thrombosis progression is prevented by anticoagulation with either low molecular weight or unfractionated heparin before transitioning to an oral anticoagulant, as well as the use of broad spectrum antibiotics. ${ }^{10}$

Identification of DST has been improved through both increased clinician awareness of the disease and improved imaging techniques. ${ }^{4} \mathrm{CTV}$, magnetic resonance imaging (MRI), magnetic resonance venography (MRV), and catheter-based cerebral angiography are the most commonly used imaging techniques to establish the diagnosis of DST. ${ }^{10} \mathrm{MRI}$ in combination with MRV is the most sensitive for clot identification." However, MRI/MRV is not routinely available as a timely study in the ED, and may be contraindicated by the presence of indwelling metallic devices such as pacemakers. Non-contrast head CT is commonly obtained as an initial imaging test in the ED for the evaluation of headache, but is frequently normal or displays non-specific findings. However, the sensitivity of CT/CTV approaches that of MRI/MRV, and is recommended as a first-line test for DST. ${ }^{3}$ Invasive cerebral angiography is the gold-standard for the diagnosis of DST, but has frequent complications and is reserved for situations where the diagnosis is equivocal after MRV or CTV. ${ }^{10}$

Ultrasound is rarely used as an imaging modality to evaluate suspected DST, due to the challenge of obtaining adequate sonographic windows to evaluate the entirety of the venous system. The use of ultrasound to investigate DST has been proposed in neonates, ${ }^{12}$ and transcranial Doppler has been reported as an indirect marker of DST..$^{13}$ Decreased flow volumes through the internal jugular vein have been demonstrated in patients with known ipsilateral DST. ${ }^{14}$ Contrast-enhanced transcranial color-coded sonography has also been proposed as a possible diagnostic approach. ${ }^{15}$ These techniques may be impractical in the ED, or beyond the skill set of most point-of-care ultrasound users.

Early identification of DST is central to ensuring favorable patient outcomes. ${ }^{16}$ We describe the use of point-of-care ultrasound to facilitate the prompt diagnosis of DST. The extra-cranial propagation of the clot permitted the treating physician to use a basic point-of-care ultrasound examination to identify a venous thrombus with intracranial extension, and leading to rapid diagnosis and treatment.

\section{CONFLICT OF INTEREST}

No potential conflict of interest relevant to this article was reported.

\section{REFERENCES}

1. Carangelo $B$, Lavalle $L$, Tiezzi $G$, et al. A rare localization of cerebral venous sinus thrombosis: case report. G Chir 2015;36: 79-83.

2. Kriss TC, Kriss VM, Warf BC. Cavernous sinus thrombophlebitis: case report. Neurosurgery 1996;39:385-9.

3. Saposnik G, Barinagarrementeria F, Brown RD Jr, et al. Diagnosis and management of cerebral venous thrombosis: a statem ent for healthcare professionals from the American Heart Association/American Stroke Association. Stroke 2011 ;42:115892.

4. Stam J. Thrombosis of the cerebral veins and sinuses. N Engl J Med 2005;352:1791-8.

5. Pongmoragot J, Saposnik G. Intracerebral hemorrhage from cerebral venous thrombosis. Curr Atheroscler Rep 2012;14: 382-9.

6. Dodick DW. Thunderclap headache. Headache 2002;42:30915.

7. Sethi P, Jones ST, Valenzuela AA. Septic cavernous sinus thrombosis with diffuse spread leading to cerebral ischemia. Orbit 2013;32:330-2.

8. Pannke TS. Cerebral dural sinus thrombosis. Ann Emerg Med 1991;20:813-6.

9. Martinelli I, Sacchi E, Landi G, Taioli E, Duca F, Mannucci PM. High risk of cerebral-vein thrombosis in carriers of a prothrombin-gene mutation and in users of oral contraceptives. N Engl J Med 1998;338:1793-7.

10. Agrawal K, Burger K, Rothrock JF. Cerebral sinus thrombosis. Headache 2016;56:1380-9.

11. Bonneville F. Imaging of cerebral venous thrombosis. Diagn Interv Imaging 2014;95:1145-50.

12. Miller E, Daneman A, Doria AS, et al. Color Doppler US of normal cerebral venous sinuses in neonates: a comparison with MR venography. Pediatr Radiol 2012;42:1070-9.

13. Canhao $P$, Batista $P$, Ferro JM. Venous transcranial Doppler in acute dural sinus thrombosis. J Neurol 1998;245:276-9.

14. Ozen 0 , Unal 0 , Avcu S. Flow volumes of internal jugular veins are significantly reduced in patients with cerebral venous sinus thrombosis. Curr Neurovasc Res 2014;11:75-82.

15. Ries S, Steinke W, Neff KW, Hennerici M. Echocontrast-enhanced transcranial color-coded sonography for the diagnosis 
Laura T. Director, et al.

of transverse sinus venous thrombosis. Stroke 1997;28:696700.

16. Ferro JM, Canhao P, Stam J, et al. Delay in the diagnosis of cerebral vein and dural sinus thrombosis: influence on outcome. Stroke 2009;40:3133-8. 